\title{
Inflammatory Bowel Disease and Patients With Mental Disorders: What Do We Know?
}

\author{
Fotios S. Fousekis ${ }^{\mathrm{a}}$, Aristeidis H. Katsanos ${ }^{\mathrm{b}}$, Georgios Kourtis ${ }^{\mathrm{c}}$, Maria Saridid ${ }^{\mathrm{d}}$, Eleni Albanie, \\ Konstantinos H. Katsanos ${ }^{\text {a }}$, Dimitrios K. Christodoulou, ${ }^{a}$
}

\begin{abstract}
Inflammatory bowel disease (IBD) is a multisystemic disease with a wide range of extraintestinal manifestations in both ulcerative colitis and Crohn's disease, while increasing evidence supports the interaction between gut and central nervous system, described as "gut-brain axis". According to epidemiological studies, it seems that patients with IBD present more frequently with impaired mental status compared to the general population, leading to diagnostic and management problems in this group of patients. The association between IBD and mental disorders, such as dementia and autism spectrum disorders, has not been fully clarified; genetic factors and the gut-brain axis seem to be involved. The purpose of this review is to present and analyze the epidemiological data about this issue, describe the possible pathogenetic mechanisms and discuss some considerations about the management of patients with IBD and impaired mental status.
\end{abstract}

Keywords: Inflammatory bowel disease; Autism; Dementia; Psychiatric diseases

\section{Introduction}

Inflammatory bowel disease (IBD) is a chronic idiopathic unknown etiology disease of gastrointestinal tract, comprising two main forms: Crohn's disease (CD) and ulcerative colitis (UC). Both disorders may also affect other organ systems as well [1]. IBD has been associated with various neurological

Manuscript submitted September 4, 2021, accepted September 20, 2021

Published online September 30, 2021

a Department of Gastroenterology, School of Health Sciences, University Hospital of Ioannina, Faculty of Medicine, University of Ioannina, Ioannina, Greece

${ }^{b}$ Division of Neurology, McMaster University, Hamilton, ON, Canada

"General Hospital "Sotiria", Athens, Greece

dUniversity of Thessaly, Greece

'University ofPatra, Patra, Greece

${ }^{\mathrm{f} C}$ Corresponding Author: Dimitrios K. Christodoulou, Department of Gastroenterology and Hepatology, School of Health Sciences, University Hospital of Ioannina, Faculty of Medicine, University of Ioannina, PO Box 1186, Ioannina 45110, Greece. Email: dchristodoulou@gmail.com

doi: https://doi.org/10.14740/jocmr4593 manifestations, such as cerebrovascular disease, demyelinating central nervous system disorder and peripheral neuropathy, indicating a gut-brain interaction in IBD [2]. In recent decade, many studies have documented a possible association between IBD and mental disorders, such as dementia, autism spectrum disorders (ASDs) and psychiatric diseases [3]. Diagnostic and Statistical Manual of Mental Disorders, 5th edition, characterizes as mental disorder, a clinical significant disturbance in an individual's cognition, emotion regulation or behavior that reflects a dysfunction in the psychological, biological or developmental processes underlying mental functioning, resulting usually in significant distress or disability in social, occupational or other important activities [4]. It is worth noting that the estimated prevalence of mental disorders among adults in the United States is approximately $20 \%$ [5], while the worldwide prevalence of autism spectrum disorders is about $1 \%$ [6].

In this narrative review, we intend to analyze the available literature regarding IBD in patients with mental disorders and discuss issues of epidemiology, pathophysiology and management in order to raise awareness and present the clinical aspects about this subgroup of IBD patients.

\section{Epidemiology}

In patients with IBD, several studies have described an increased frequency of various mental disorders, ranging from mild depression to severe form of schizophrenia and serious dementia, while the prevalence of mental disorders depends on the study (Table 1) [7-21].

\section{Psychiatric diseases}

Many studies have described that IBD patients often suffer from anxiety and depression that may influence the course of disease through the gut-brain axis [22]. A recent systematic review demonstrated that the prevalence of anxiety and depression is higher in patients with IBD (anxiety $19.1 \%$ versus $9.6 \%$ and depression $21.2 \%$ versus $13.4 \%$ ), while the rates seem to be higher when the disease is active [7]. Risk factors of depression among patients with IBD seem to be age, severe disease, flares, disable or unemployed status, socioeconomic deprivation [23] and coronavirus disease 2019 (COVID-19) lockdown [24]. Furthermore, several studies have demonstrated that the rate of suicidal ideation seems to be increased in IBD patients 
Table 1. Mental Disorders Associated With Inflammatory Bowel Disease

\begin{tabular}{l}
\hline Psychiatric diseases \\
Depression [7] \\
Bipolar disorder [8,9] \\
Schizophrenia [10] \\
Dementia \\
Alzheimer's disease $[14,15]$ \\
Parkinson's disease [16] \\
Vascular dementia [20, 21] \\
Autism spectrum disorders [11-13] \\
Genetic syndromes \\
Down syndrome [17] \\
Turner syndrome [18] \\
Williams syndrome [19]
\end{tabular}

and is approximately $6 \%$ [25].

Regarding the association between bipolar disorder and IBD, the data are conflicting. A population-based IBD cohort study found lower rates of bipolar disorder in IBD patients than in control subjects in the United States and New Zeeland [26]. However, a population-based cross-sectional study from Taiwan demonstrated that patients with IBD were more likely to have bipolar disorder (adjusted odds ratio (OR): 2.1; $95 \%$ confidence interval (CI): 1.30 - 3.38) [8], while a national cohort study from Denmark found higher risk of bipolar disorder only in patients with CD [9]. In addition, a Canadian cohort study demonstrated a higher incidence rate ratio of schizophrenia in IBD patients compared to matched-control individuals to age and sex, but this was not statistically significant (1.64; 95\% CI: 0.95 - 2.84) [10]. Also, a general population-based study from United Kingdom demonstrated no evidence of an increased likelihood of schizophrenia in patients with $\mathrm{CD}$ (OR: 0.74; 95\% CI: $0.44-1.3$ ) and UC (OR: 0.71; 95\% CI: 0.44 - 1.1) in comparison with the general population [27].

Some case reports and case series have suggested a possible association between eating disorders, such as bulimia nervosa and anorexia, and IBD; however, the evidence is scarce and most studies are required in order to support this causal association [28].

\section{ASDs}

According to the Diagnostic and Statistical Manual of Mental Disorders, 5th edition, ASDs are characterized by deficits in social communications and the presence of restricted interests and repetitive behaviors, including four separate disorders: autistic disorder, Asperger's syndrome, childhood disintegrative disorder and the catch-all diagnosis of pervasive developmental disorder not otherwise specified [29]. Many studies have demonstrated that children with ASD suffer frequently from gastrointestinal symptoms, such as diarrhea, constipa- tion, abdominal pain or gaseousness, mainly children with ASD and increased problem behaviors, such as social withdrawal, sleep problems and self-injuries [30,31]. According to a meta-analysis, children with ASD have three times higher risk of gastrointestinal symptoms compared to children without ASD [32].

Recently, four case-control study populations have documented higher prevalence of IBD among patients with ASD than that of their respective controls [11]. Furthermore, a population-based study of more than 48,000 children with ASD demonstrated a higher prevalence of $\mathrm{CD}$ and $\mathrm{UC}$ in children with ASD compared to controls ( $0.18 \%$ vs. $0.11 \%)$. Also, this study demonstrated that children with ASD and IBD received more frequently second-tier biological agents, such as adalimumab and certolizumab, compared to pediatric IBD population without ASD. These findings may have two possible explanations. Children with ASD and IBD may suffer from more severe IBD or IBD refractory to first-line biological agents, such as infliximab. The second explanation is that children with ASD due to their behavior present less compliance and adherence to oral and intravenous treatment, making subcutaneous treatment more feasible [12]. In addition, a retrospective study documented an increased prevalence of IBD in hospitalized patients with ASD compared to the overall hospital population in four hospitals in Boston $(0.83 \%$ vs. $0.54 \%)[13]$.

\section{Dementia}

Dementia is a heterogeneous syndrome that may be caused by many different neurological diseases. The major cause of dementia is neurodegenerative disorders, such as Alzheimer disease, Lewy bodies dementia and Parkinson's disease, while the estimated global prevalence is approximately $7 \%$ of individuals 65 years or older [33]. A recent population-based cohort study demonstrated an overall higher incidence of dementia among patients with IBD (5.5\% vs. $1.4 \%$ among controls). In addition, dementia was diagnosed at an earlier age among patients with IBD (76.24 years vs. 83.45 years) and the risk ratio of developing dementia among patients with IBD was 2.54 (95\% CI: 1.91 - 3.37). Furthermore, the risk for dementia was associated with the chronicity of IBD diagnosis and dementia risk seems to accelerate over time correlating with the chronicity of IBD diagnosis, while no significant differences in the risk for dementia were demonstrated between patients with $\mathrm{UC}$ and $\mathrm{CD}[14]$.

Concerning Parkinson disease, dementia occurs in 30\% of patients with Parkinson's disease [15], while several epidemiological studies have demonstrated an association between Parkinson's disease and IBD [34]. A recent meta-analysis found an overall increased risk of Parkinson's disease development compared to control individuals (risk ratio (RR): $1.41 ; 95 \%$ CI: 1.19 - 1.66). Patients with CD had a $28 \%$ increased risk of Parkinson's disease (RR: 1.28; 95\% CI: 1.08 - 1.52) and UC patients had a 30\% increased risk of Parkinson's disease (RR: $1.30 ; 95 \%$ CI: 1.15 - 1.47) [16]. In addition, many studies have demonstrated that IBD treatment seems to decrease the subsequent risk of Parkinson's disease development [35]. Instead, 


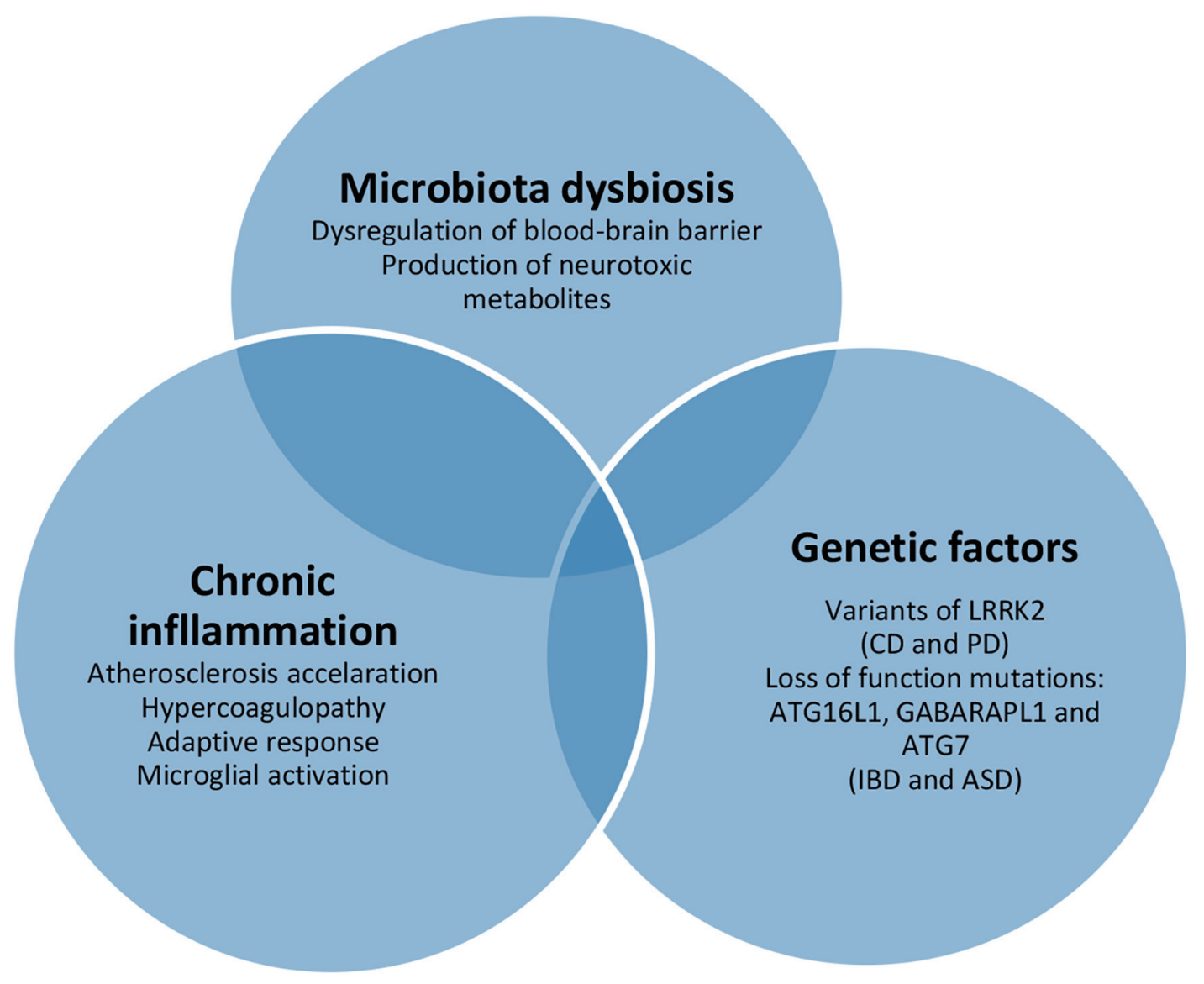

Figure 1. Inflammatory bowel disease-associated pathogenetic mechanisms of mental disorders. CD: Crohn's disease; PD: Parkinson's disease; IBD: inflammatory bowel disease; ASD: autism spectrum disorder.

patients with Parkinson's disease seem to have no higher risk for either CD or UC [36].

\section{Genetic syndromes with impaired intellectual ability}

Several case reports have described IBD development in patients with genetic syndromes that cause intellectual disability, such as Down syndrome [17], Turner syndrome [18] and Williams syndrome [19]; however, data are very limited and no association has been documented.

\section{Pathogenesis}

Although the pathogenetic mechanisms between IBD and mental disorders have not been fully elucidated, genetic factors and the gut-brain interaction seem to contribute to the elevated prevalence of mental disorders in patients with IBD (Fig. 1).

\section{Psychiatric diseases}

Several inflammatory diseases, including IBD, have been as- sociated with anxiety and depression development. Clinical studies have demonstrated that cytokine release may affect neurobehavioral functions and cause negative effects on emotional and memory effects $[37,38]$. Also, animal models have shown that inflammation induction may cause hypothalamicpituitary-adrenal axis dysregulation and changes in bloodbrain barrier integrity. The disruption of blood-brain barrier leads to increased inflammatory mediators in central neural system (CNS), affecting the neurotransmitter metabolism, in brain regions associated with emotional regulation, such as the nucleus accumbens, amygdale and hippocampus [39]. Furthermore, some authors have suggested that depression in inflammatory conditions is a form or a consequence of sickness behavior and it is an adaptive response that enhances recovery by conserving energy to combat acute inflammation [40].

On the other hand, it should be mentioned that psychological factors may affect the course of IBD and several studies have described the role of psychological factors on the course of IBD, demonstrating that psychological stress may cause disease relapse or exacerbation [41]. Stress may induce changes in gastrointestinal function, such as increased intestinal motility and permeability, and activation mucosal mast cells, from which the course of IBD may be influenced $[42,43]$. 
Table 2. Management of Patients With Inflammatory Bowel Disease and Mental Disorder

Collaboration between gastroenterologists, neurologists, psychiatrists and patient's family members

Deep sedation during endoscopy

Subcutaneous biological agents might be preferred due to better compliance

Avoid corticosteroid treatment

Second-generation corticosteroids could be preferred

Physical activity

Avoid alcohol and narcotics use

\section{ASDs}

Genetic and immunological factors are included to the possible explanations of the epidemiological association between IBD and ASD. A study demonstrated that two losses of function mutations previously implicated in IBD on the autophagy pathway (ATG16L1 and GABARAPL1) may play a role in ASD development, while one ASD-associated loss of function mutation (ATG7) may contribute to IBD [44]. Furthermore, identification of an overlap gene expression in gastrointestinal tissue in pediatric patients with IBD and in children with ASD has been described [45]. In addition, gut microbiome may participate in association between IBD and ASD, as individuals with ASD seem to have an altered gut microbiota [46] and microbiota dysbiosis may contribute to IBD development [47]. Further studies are required due to the lack of data about this topic.

\section{Dementia}

The pathogenic mechanism of the increased risk of dementia in patients with IBD may be multifactorial and involves chronic inflammation, atherosclerosis acceleration and hypercoagulopathy. In particular, Alzheimer's disease is the most frequent cause of dementia and the major pathogenetic mechanisms include the development of amyloid $\beta$ plaques and neurofibrillary tangles [48]. Several studies have suggested that systemic chronic inflammation may accelerate the progression of Alzheimer's disease via alterations of the blood-brain barrier and the microglial activation [49], indicating that IBD as a chronic inflammatory condition may contribute to Alzheimer's disease progression.

Regarding Parkinson's disease, mutations of leucine-rich repeat kinase 2 (LRRK-2) seem to cause disrupted autophagy and participate in Parkinson's disease development [50], as a recent study identified shared genetic variants of LRRK-2 between Parkinson's disease and CD [51]. Furthermore, gutbrain axis may contribute to Parkinson's disease development in patients with IBD, as animal studies have suggested that alterations of gut microbiota may trigger Parkinson's disease [52], while a recent study demonstrated that gastrointestinal infections are associated with a higher risk of Parkinson's disease [53].

In addition, IBD has been associated with a higher risk of thromboembolic events and stroke development due to thrombocytosis, coagulation abnormalities, hyperlipidemia and hyperhomocysteinemia [20], thus contributing to the development of vascular dementia. It is worth mentioning that vascular dementia seems to cause approximately $15 \%$ of dementia cases [21].

Furthermore, dysbiosis of the gut microbiome seems to be present in patients with IBD [54] and many studies have suggested that dysregulation of the gut microbiota may play a role in the progression of dementia through alterations of the gut barrier, production of microbial neurotoxic metabolites in systemic circulation and increased inflammation $[55,56]$.

\section{Management}

Treatment and management of patients with IBD and mental illness poses many serious problems and is often a challenge due to difficulties in compliance, communication problems and non-cooperation in the physical examination of this group of patients (Table 2). We suggest that collaboration between gastroenterologists, psychiatrists and patient's family members may be beneficial and helpful in treatment compliance and monitoring of these patients. For the aforementioned reasons, diagnosis of IBD in patients with mental disorders may be difficult, while endoscopy may be a complex process or even not feasible, especially in patients with severe dementia or major psychiatric disorders. Deep sedation may be mandatory during endoscopy, while adequated bowel preparation for colonoscopy remains a significant issue. In addition, the choice of the appropriate treatment should take into account several aspects. The use of intravenous biological agents requires inpatient treatment, while azathioprine use requires close monitoring due to frequent adverse events, mainly within the first weeks. Furthermore, we would recommend avoiding the frequent use of corticosteroids in patients with psychiatric disorders. Many studies have demonstrated corticosteroid-induced psychiatric side effects, including disturbances of cognition, mood, sleep and behavior through implication of the hypothalamicpituitary-adrenal axis. The estimated prevalence of psychiatric side effects ranges from $1 \%$ to $10 \%$, while dosage and duration of corticosteroids administration are directly related to the frequency of adverse effects $[57,58]$. In a recent prospective study, the rate of corticosteroid-induced mood changes in IBD patients after 2 weeks of prednisone $40 \mathrm{mg}$ daily was $49.1 \%$ [59]. In the last decade, second generation of topical active corticosteroids, such as budesonide and beclomethasone, are 
often used in UC and CD treatment and have been associated with high efficacy, presenting fewer adverse events through their low systemic bioavailability and approximately $90 \%$ first-pass metabolism in liver [60]. On the other hand, according to available data, treatment with biological agents does not seem to be associated with an increased risk of anxiety, depression, suicidality and psychosis [61]. Thus, the use of subcutaneous biological agents, such as adalimumab, golimumab and ustekinumab, in patients with mental illness may be a safe, effective and feasible treatment.

Regarding the impact of thiopurine on clinical course of mental disorders in IBD patients, there are conflicting data. Thiopurines seem to inhibit the Ras-related C3 botulinum substrate (Rac 1), which has been implicated in Alzheimer's disease development via increased amyloid precursor protein [62]. A recent national cohort study from United States described that IBD patients receiving thiopurine have a lower risk of Alzheimer's disease compared with IBD patients without thiopurine exposed, demonstrating an adjusted hazard ratio (HR) per additional year of thiopurine exposure 0.917 (95\% CI: $0.851-0.989$ ) for comorbidities and medications [63]. On the other hand, a retrospective cohort study demonstrated that IBD patients exposed to thiopurine less than 1 year had a $67 \%$ higher risk of depression (HR: 1.667; 95\% CI: $1.501-1.805$ ) compared to IBD patients without thiopurine exposure [64]. However, prospective studies and further researches into the effect of thiopurines in IBD patients on neuro-psychiatric disorders are required.

As mentioned above, patients with IBD appear to have a higher risk of dementia, which is prevalent in the elderly. It is estimated that $10-30 \%$ of patients with IBD are $>60$ years old. Elderly patients may have less complicated clinical course; however, they have higher or similar rates of surgery [65]. In addition, elderly patients tent to have more frequent comorbidities, such as heart disease and diabetes, which may affect the management and clinical course of disease. IBD patients with diabetes may have an increased risk of flare and IBD-related hospitalization [66], while anti-TNF agents are contraindicated in patients with severe heart failure [67]. Consequently, an individualized care of IBD patients with dementia is required.

Furthermore, it is worth mentioning that increased clinicians' awareness for the development of dementia and psychiatric disorders in patients with IBD is warranted to facilitate an early diagnosis and prompt the appropriate treatment initiation. In addition, because of high prevalence of irritable bowel syndrome (IBS) in patients with psychiatric disorders and ASDs [68], clinicians should distinguish IBD from IBS. IBS is a functional disorder, characterized by chronic abdominal pain and altered bowel habits. Patients with IBS report at least once weekly abdominal pain in association with a change in stool form, frequency and/or abdominal pain related to defecation, while IBS diagnosis requires exclusion of disorders, such as IBD and celiac disease [69]. Likewise, symptoms indicating IBD, such as bloody stools, malabsorption, chronic diarrhea and iron deficiency, in patients with mental disorders should be evaluated in detail and endoscopy should be considered.

Lifestyle modifications may have a significant role in IBD and mental disorders. Patients with IBD should be encouraged to exercise because physical activity may improve psycho- logical well-being, reducing anxiety and depression, as well as having a preventative or mitigated role against Alzheimer's disease [70], Parkinson's disease [71] and ASD [72]. Also, IBD patients should avoid alcohol consumption and narcotics use that have been correlated with comorbid mental illnesses and worsening of IBD course [73].

\section{Conclusions}

The prevalence of disorders with impaired mental status, such as psychiatric diseases, ASDs and dementia seems to be higher in patients with IBD compared to the general population. The pathogenetic mechanisms may be multifactorial, including alteration of gut microbiota, genetic factors and chronic inflammation. The diagnosis of IBD and management of this group of IBD patients is often challenging due to poor compliance and a close collaboration between a team of gastroenterologists, neurologists, psychiatrists and family members of the patient is required.

\section{Acknowledgments}

None to declare.

\section{Financial Disclosure}

No funding has been received.

\section{Conflict of Interest}

All authors declare no conflict of interest.

\section{Author Contributions}

DKC and KHK conceived designed and critically revised the article for important intellectual content. FSF, GK, MS, and EA contributed to the data collection and literature research. FSF and AHK analyzed the data and contributed substantially in the manuscript preparations. All authors approved the final version of the article to be published.

\section{Data Availability}

The authors declare that data supporting the findings of this study are available within the article.

\section{References}

1. Vavricka SR, Schoepfer A, Scharl M, Lakatos PL, Navarini A, Rogler G. Extraintestinal manifestations of inflammatory bowel disease. Inflamm Bowel Dis. 
2015;21(8):1982-1992.

2. Moris G. Inflammatory bowel disease: an increased risk factor for neurologic complications. World J Gastroenterol. 2014;20(5):1228-1237.

3. Nowakowski J, Chrobak AA, Dudek D. Psychiatric illnesses in inflammatory bowel diseases - psychiatric comorbidity and biological underpinnings. Psychiatr Pol. 2016;50(6):1157-1166.

4. Telles-Correia D. Mental disorder: Are we moving away from distress and disability? J Eval Clin Pract. 2018;24(5):973-977.

5. McGinty EE, Daumit GL. Integrating mental health and addiction treatment into general medical care: the role of policy. Psychiatr Serv. 2020;71(11):1163-1169.

6. Lai MC, Lombardo MV, Baron-Cohen S. Autism. Lancet. 2014;383(9920):896-910.

7. Mikocka-Walus A, Knowles SR, Keefer L, Graff L. Controversies revisited: a systematic review of the comorbidity of depression and anxiety with inflammatory bowel diseases. Inflamm Bowel Dis. 2016;22(3):752-762.

8. Kao LT, Lin HC, Lee HC. Inflammatory bowel disease and bipolar disorder: A population-based cross-sectional study. J Affect Disord. 2019;247:120-124.

9. Eaton WW, Pedersen MG, Nielsen PR, Mortensen PB. Autoimmune diseases, bipolar disorder, and non-affective psychosis. Bipolar Disord. 2010;12(6):638-646.

10. Bernstein CN, Hitchon CA, Walld R, Bolton JM, Sareen J, Walker JR, Graff LA, et al. Increased burden of psychiatric disorders in inflammatory bowel disease. Inflamm Bowel Dis. 2019;25(2):360-368.

11. Doshi-Velez F, Avillach P, Palmer N, Bousvaros A, Ge Y, Fox K, Steinberg G, et al. Prevalence of inflammatory bowel disease among patients with autism spectrum disorders. Inflamm Bowel Dis. 2015;21(10):2281-2288.

12. Lee M, Krishnamurthy J, Susi A, Sullivan C, Gorman GH, Hisle-Gorman E, Erdie-Lalena CR, et al. Association of autism spectrum disorders and inflammatory bowel disease. J Autism Dev Disord. 2018;48(5):1523-1529.

13. Kohane IS, McMurry A, Weber G, MacFadden D, Rappaport L, Kunkel L, Bickel J, et al. The co-morbidity burden of children and young adults with autism spectrum disorders. PLoS One. 2012;7(4):e33224.

14. Zhang B, Wang HE, Bai YM, Tsai SJ, Su TP, Chen TJ, Wang YP, et al. Inflammatory bowel disease is associated with higher dementia risk: a nationwide longitudinal study. Gut. 2021;70(1):85-91.

15. Hanagasi HA, Tufekcioglu Z, Emre M. Dementia in Parkinson's disease. J Neurol Sci. 2017;374:26-31.

16. Zhu F, Li C, Gong J, Zhu W, Gu L, Li N. The risk of Parkinson's disease in inflammatory bowel disease: A systematic review and meta-analysis. Dig Liver Dis. 2019;51(1):38-42.

17. Souto-Rodriguez R, Barreiro-de-Acosta M, DominguezMunoz JE. Down's syndrome and inflammatory bowel disease: is there a real link? Rev Esp Enferm Dig. 2014;106(3):220-222.

18. Hyodo H, Tomita Y, Hirai K, Hirakawa H, Ueno S, Ishiguro H. Turner syndrome with ulcerative colitis. Clin Pediatr Endocrinol. 2009;18(4):101-105.
19. Gilbert-Barness E, Fox T, Morrow G, Luquette M, Pomerance HH. Williams syndrome associated with Crohn disease, multiple infections, and chronic granulomatous disease. Fetal Pediatr Pathol. 2004;23(1):29-37.

20. Katsanos AH, Kosmidou M, Giannopoulos S, Katsanos KH, Tsivgoulis G, Kyritsis AP, Tsianos EV. Cerebral arterial infarction in inflammatory bowel diseases. Eur J Intern Med. 2014;25(1):37-44.

21. O'Brien JT, Thomas A. Vascular dementia. Lancet. 2015;386(10004):1698-1706.

22. Sajadinejad MS, Asgari K, Molavi H, Kalantari M, Adibi P. Psychological issues in inflammatory bowel disease: an overview. Gastroenterol Res Pract. 2012;2012:106502.

23. Nahon S, Lahmek P, Durance C, Olympie A, Lesgourgues B, Colombel JF, Gendre JP. Risk factors of anxiety and depression in inflammatory bowel disease. Inflamm Bowel Dis. 2012;18(11):2086-2091.

24. Sempere L, Bernabeu P, Cameo J, Gutierrez A, Laveda R, Garcia MF, Aguas M, et al. Evolution of the emotional impact in patients with early inflammatory bowel disease during and after Covid-19 lockdown. Gastroenterol Hepatol. 2021.

25. Santa A, Szanto KJ, Sarlos P, Miheller P, Farkas K, Molnar T. Letter: suicide risk among adult inflammatory bowel disease patients. Aliment Pharmacol Ther. 2020;51(11):1213-1214.

26. Walker JR, Ediger JP, Graff LA, Greenfeld JM, Clara I, Lix L, Rawsthorne P, et al. The Manitoba IBD cohort study: a population-based study of the prevalence of lifetime and 12-month anxiety and mood disorders. Am J Gastroenterol. 2008;103(8):1989-1997.

27. West J, Logan RF, Hubbard RB, Card TR. Risk of schizophrenia in people with coeliac disease, ulcerative colitis and Crohn's disease: a general population-based study. Aliment Pharmacol Ther. 2006;23(1):71-74.

28. Ilzarbe L, Fabrega M, Quintero R, Bastidas A, Pintor L, Garcia-Campayo J, Gomollon F, et al. Inflammatory bowel disease and eating disorders: a systematized review of comorbidity. J Psychosom Res. 2017;102:47-53.

29. Hodges H, Fealko C, Soares N. Autism spectrum disorder: definition, epidemiology, causes, and clinical evaluation. Transl Pediatr. 2020;9(Suppl 1):S55-S65.

30. Chaidez V, Hansen RL, Hertz-Picciotto I. Gastrointestinal problems in children with autism, developmental delays or typical development. J Autism Dev Disord. 2014;44(5):1117-1127.

31. Restrepo B, Angkustsiri K, Taylor SL, Rogers SJ, Cabral J, Heath B, Hechtman A, et al. Developmental-behavioral profiles in children with autism spectrum disorder and co-occurring gastrointestinal symptoms. Autism Res. 2020;13(10):1778-1789.

32. McElhanon BO, McCracken C, Karpen S, Sharp WG. Gastrointestinal symptoms in autism spectrum disorder: a meta-analysis. Pediatrics. 2014;133(5):872-883.

33. Gale SA, Acar D, Daffner KR. Dementia. Am J Med. 2018;131(10):1161-1169.

34. Brudek T. Inflammatory bowel diseases and Parkinson's disease. J Parkinsons Dis. 2019;9(s2):S331-S344.

35. Lee HS, Lobbestael E, Vermeire S, Sabino J, Cleynen 
I. Inflammatory bowel disease and Parkinson's disease: common pathophysiological links. Gut. 2021;70(2):408417.

36. Camacho-Soto A, Gross A, Searles Nielsen S, Dey N, Racette BA. Inflammatory bowel disease and risk of Parkinson's disease in Medicare beneficiaries. Parkinsonism Relat Disord. 2018;50:23-28.

37. Reichenberg A, Yirmiya R, Schuld A, Kraus T, Haack M, Morag A, Pollmacher T. Cytokine-associated emotional and cognitive disturbances in humans. Arch Gen Psychiatry. 2001;58(5):445-452.

38. Grigoleit JS, Kullmann JS, Wolf OT, Hammes F, Wegner A, Jablonowski S, Engler H, et al. Dose-dependent effects of endotoxin on neurobehavioral functions in humans. PLoS One. 2011;6(12):e28330.

39. Abautret-Daly A, Dempsey E, Parra-Blanco A, Medina C, Harkin A. Gut-brain actions underlying comorbid anxiety and depression associated with inflammatory bowel disease. Acta Neuropsychiatr. 2018;30(5):275-296.

40. Maes M, Berk M, Goehler L, Song C, Anderson G, Galecki P, Leonard B. Depression and sickness behavior are Janus-faced responses to shared inflammatory pathways. BMC Med. 2012;10:66.

41. Maunder RG, Levenstein S. The role of stress in the development and clinical course of inflammatory bowel disease: epidemiological evidence. Curr Mol Med. 2008;8(4):247-252.

42. Hollander D. Inflammatory bowel diseases and brain-gut axis. J Physiol Pharmacol. 2003;54(Suppl 4):183-190.

43. Theoharides TC, Cochrane DE. Critical role of mast cells in inflammatory diseases and the effect of acute stress. J Neuroimmunol. 2004;146(1-2):1-12.

44. Somekh J, Peleg M, Eran A, Koren I, Feiglin A, Demishtein A, Shiloh R, et al. A model-driven methodology for exploring complex disease comorbidities applied to autism spectrum disorder and inflammatory bowel disease. J Biomed Inform. 2016;63:366-378.

45. Walker SJ, Fortunato J, Gonzalez LG, Krigsman A. Identification of unique gene expression profile in children with regressive autism spectrum disorder (ASD) and ileocolitis. PLoS One. 2013;8(3):e58058.

46. Vuong HE, Hsiao EY. Emerging roles for the gut microbiome in autism spectrum disorder. Biol Psychiatry. 2017;81(5):411-423.

47. Qi Y, Wu HM, Yang Z, Zhou YF, Jin L, Yang MF, Wang FY. New insights into the role of oral microbiota dysbiosis in the pathogenesis of inflammatory bowel disease. Dig Dis Sci. 2021.

48. Tiwari S, Atluri V, Kaushik A, Yndart A, Nair M. Alzheimer's disease: pathogenesis, diagnostics, and therapeutics. Int J Nanomedicine. 2019;14:5541-5554.

49. Calsolaro V, Edison P. Neuroinflammation in Alzheimer's disease: Current evidence and future directions. Alzheimers Dement. 2016;12(6):719-732.

50. Alessi DR, Sammler E. LRRK2 kinase in Parkinson's disease. Science. 2018;360(6384):36-37.

51. Hui KY, Fernandez-Hernandez H, Hu J, Schaffner A, Pankratz N, Hsu NY, Chuang LS, et al. Functional variants in the LRRK2 gene confer shared effects on risk for
Crohn's disease and Parkinson's disease. Sci Transl Med. 2018;10(423):eaai7795.

52. Matheoud D, Cannon T, Voisin A, Penttinen AM, Ramet L, Fahmy AM, Ducrot C, et al. Intestinal infection triggers Parkinson's disease-like symptoms in Pink1(-/-) mice. Nature. 2019;571(7766):565-569.

53. Nerius M, Doblhammer G, Tamguney G. GI infections are associated with an increased risk of Parkinson's disease. Gut. 2020;69(6):1154-1156.

54. Ni J, Wu GD, Albenberg L, Tomov VT. Gut microbiota and IBD: causation or correlation? Nat Rev Gastroenterol Hepatol. 2017;14(10):573-584.

55. Alkasir R, Li J, Li X, Jin M, Zhu B. Human gut microbiota: the links with dementia development. Protein Cell. 2017;8(2):90-102.

56. Saji N, Murotani K, Hisada T, Kunihiro T, Tsuduki T, Sugimoto T, Kimura A, et al. Relationship between dementia and gut microbiome-associated metabolites: a cross-sectional study in Japan. Sci Rep. 2020;10(1):8088.

57. Warrington TP, Bostwick JM. Psychiatric adverse effects of corticosteroids. Mayo Clin Proc. 2006;81(10):13611367.

58. Kenna HA, Poon AW, de los Angeles CP, Koran LM. Psychiatric complications of treatment with corticosteroids: review with case report. Psychiatry Clin Neurosci. 2011;65(6):549-560.

59. Ou G, Bressler B, Galorport C, Lam E, Ko HH, Enns $\mathrm{R}$, Telford J, et al. Rate of corticosteroid-induced mood changes in patients with inflammatory bowel disease: a prospective study. J Can Assoc Gastroenterol. 2018;1(3):99-106.

60. Salice M, Rizzello F, Calabrese C, Privitera Hrustemovic H, Gionchetti P. Budesonide MMX: efficacy and safety profile in the treatment of ulcerative colitis. Expert Rev Gastroenterol Hepatol. 2019;13(7):607-613.

61. Jain A, Marrie RA, Shafer LA, Graff LA, Patten SB, ElGabalawy R, Sareen J, et al. Incidence of adverse psychiatric events during treatment of inflammatory bowel disease with biologic therapies: a systematic review. Crohns Colitis 360. 2020;2(1):otz053.

62. Wang PL, Niidome T, Akaike A, Kihara T, Sugimoto H. Rac1 inhibition negatively regulates transcriptional activity of the amyloid precursor protein gene. J Neurosci Res. 2009;87(9):2105-2114

63. Sutton SS, Magagnoli J, Cummings T, Hardin JW. Association between thiopurine medication exposure and Alzheimer's disease among a cohort of patients with inflammatory bowel disease. Alzheimers Dement (N Y). 2019;5:809-813.

64. Sutton SS, Magagnoli J, Cummings T, Hardin JW, Love BL. Association between thiopurine exposure and depression in patients with inflammatory bowel disease and rheumatoid arthritis. J Psychopharmacol. 2020;34(10):11631167.

65. Ananthakrishnan AN, Shi HY, Tang W, Law CC, Sung JJ, Chan FK, Ng SC. Systematic review and meta-analysis: phenotype and clinical outcomes of older-onset inflammatory bowel disease. J Crohns Colitis. 2016;10(10):12241236. 
66. Kumar A, Teslova T, Taub E, Miller JD, Lukin DJ. Comorbid diabetes in inflammatory bowel disease predicts adverse disease-related outcomes and infectious complications. Dig Dis Sci. 2021;66(6):2005-2013.

67. Ananthakrishnan AN, Donaldson T, Lasch K, Yajnik V. Management of inflammatory bowel disease in the elderly patient: challenges and opportunities. Inflamm Bowel Dis. 2017;23(6):882-893.

68. Fadgyas-Stanculete M, Buga AM, Popa-Wagner A, Dumitrascu DL. The relationship between irritable bowel syndrome and psychiatric disorders: from molecular changes to clinical manifestations. J Mol Psychiatry. 2014;2(1):4.

69. Lacy BE, Pimentel M, Brenner DM, Chey WD, Keefer LA, Long MD, Moshiree B. ACG clinical guideline: management of irritable bowel syndrome. Am J Gastro- enterol. 2021;116(1):17-44.

70. Gronek P, Balko S, Gronek J, Zajac A, Maszczyk A, Celka R, Doberska A, et al. Physical activity and Alzheimer's disease: a narrative review. Aging Dis. 2019;10(6):12821292.

71. Fayyaz M, Jaffery SS, Anwar F, Zil-E-Ali A, Anjum I. The effect of physical activity in Parkinson's disease: a mini-review. Cureus. 2018.

72. Huang J, Du C, Liu J, Tan G. Meta-analysis on intervention effects of physical activities on children and adolescents with autism. Int J Environ Res Public Health. 2020;17(6):1950.

73. Mantzouranis G, Fafliora E, Saridi M, Tatsioni A, Glanztounis G, Albani E, Katsanos KH, et al. Alcohol and narcotics use in inflammatory bowel disease. Ann Gastroenterol. 2018;31(6):649-658. 